\title{
Proceso evaluativo del material cartográfico temático táctil para alumnos con discapacidad visual y auditiva
}

\author{
Teresa Barrientos Guzmán* \\ Enrique Pérez de Prada**
}

\begin{abstract}
The visually and hearing impaired users represent a special universe around the world, who need access to information, in this specific case information that is related to environmental problems and their manifestation in various regional processes such as melting glaciers among others.

The power to represent the spatial relationships between humans and their natural environment is a major challenge with which to achieve the formation of the image mental perception of the average real places and problems. The map helps this as it is an abstraction of what happens in reality in the environmental area, their features and their distributions.

All tactile map product adaptation involves one visual map to provide information by touch, step that involves a series of steps that are basic recitals to make a tactile mapping, where the end-user features are a major factor importance.

In this process, the evaluation of map products is fundamental because it allows us to check on adaptation achieved its goal of providing information to people who are blind or deaf, if they differ not only textures, shapes, colors between other aspects also seeks to determine whether the user-understood conceptual part of each of the representations.
\end{abstract}

Key words: Evaluation process tactile cartography, evaluator, physical, conceptual aspects, tailored maps, thematic maps touch.

* Correo electrónico: teresabarrientos@vtr.net

**Correo electrónico: eperez@utem.cl 


\section{Resumen}

Los usuarios discapacitados visuales y auditivos representan un universo especial en todo el mundo, que necesitan tener acceso a la información, en este caso específico a información que está relacionada con la problemática ambiental y su manifestación en diversos procesos territoriales como es el deshielo de los glaciares entre otros.

El poder representar las relaciones espaciales entre el ser humano y su entorno natural es un desafío primordial con el cual se puede lograr la formación de la imagen mental con la percepción de los lugares del medio real y sus problemáticas. El mapa ayuda a esto ya que es una abstracción de lo que sucede en la realidad en el área ambiental, sus fenómenos y sus distribuciones.

Todo producto cartográfico táctil involucra la adaptación del mapa visual a uno que comunique la información por medio del tacto, etapa que involucra una serie de pasos que son los considerandos básicos al hacer una cartografía táctil, donde las características del usuario final son un factor de gran importancia.

En este proceso, la evaluación de los productos cartográficos es un aspecto fundamental, ya que nos permite comprobar si la adaptación realizada logró su objetivo de comunicar la información a las personas ciegas o sordas, no sólo si se diferencian las texturas, formas, colores entre otros aspectos, además se busca determinar si el usuario comprendió la parte conceptual de cada una de las representaciones.

Palabras claves: Proceso evaluativo, cartografía táctil, evaluador, aspectos físicos, aspectos conceptuales, mapas adaptados, mapas temáticos táctiles.

\section{Introducción}

Una característica importante que tienen los mapas, es que son medios preponderantes para el almacenamiento y comunicación de información sobre la localización y caracterización del mundo natural, de la sociedad y la cultura y en este caso especial, de la problemática del calentamiento global. A través de los mapas podemos reconocer la distribución y relaciones espaciales de los fenómenos geográficos, que expresan esta problemática, y los cambios que se están produciendo a causa de las alteraciones climáticas que se manifiestan en distintas partes de nuestro planeta.

En el caso de las personas ciegas, por medio de puntos de referencia, pueden hacer su propio mapa mental del espacio que está siendo afectado por estos cambios. Es imprescindible tener en cuenta cómo las personas ciegas y sordas obtienen información para lograr generar su mapa mental, donde la descripción oral, la visita a lugares y los mapas táctiles se constituyen en una fuente primordial de información. 
La cartografía táctil es la que más aporta a las personas ciegas y sordas en la comprensión del espacio geográfico y de los fenómenos que en éste se desarrollen, ya que utiliza la representación en relieve por medio de texturas, líneas y formas, "ésta al contrario que la cartografía visual, es una forma de comunicación secuencial, como lo es la escritura, nadie lee una página de un vistazo, tiene que ir palabra por palabra hasta comprender la información que la página contiene, en un mapa táctil sucede lo mismo, mientras una persona vidente descubre toda la información de una forma instantánea (tal y como llega a su cerebro), los lectores de mapas visualmente incapacitados deben descubrir la información mediante un barrido secuencial del mapa" (Maestro I., 2004).

En este sentido los usuarios discapacitados visuales y auditivos son una población especial, que necesitan tener acceso a datos e información que les permitan estructurar sus propios mapas mentales (Coll, 2003), por lo cual se transforma en un objetivo primordial, tener la certeza que con estos productos cartográficos se está logrando comunicar la problemática ambiental.

Esta iniciativa considera la creación de un material inclusivo, permitiendo acceder también a él, a niños y/o jóvenes ciegos y sordos, donde puedan relacionarse con esta problemática en igualdad de condiciones que sus compañeros de aula.

Para realizar los productos cartográficos táctiles que fueron evaluados, fue necesario investigar especialmente en cuanto a la mejor forma de representación de la información, donde se utilizan los símbolos tanto puntuales, lineales y areales, los cuales pueden transmitir la realidad, considerando que es muy difícil y complejo adaptarlos a un sistema táctil, donde la abstracción es una variable fundamental a considerar para lograr representar las mismas regularidades y las mismas recurrencias que sustentan a los fenómenos geográficos (Benoit et al., 1993).

De lo planteado anteriormente nace la inquietud, ¿se está logrando el objetivo de comunicar eficientemente la problemática ambiental y sus consecuencias por medio de los productos generados? La respuesta sólo la podemos obtener por medio de la aplicación de instrumentos de evaluación del material desarrollado y su posterior tabulación.

\section{Objetivos}

1. Determinar por medio del proceso de evaluación, si se está logrando comunicar la problemática ambiental y sus consecuencias a través de los productos temáticos táctiles.

2. Determinar las instancias más adecuadas para aplicar los test de evaluación de los productos temáticos táctiles generados en el área de la problemática ambiental, para personas con discapacidad sensorial. 


\section{Proceso de evaluación}

La construcción de un producto cartográfico táctil siempre incluye un proceso arduo de adaptación del mapa visual para transformarlo en un mapa que comunique también la información a través del tacto, lo cual implica:

- Analizar bases cartográficas para encontrar aquella que cumpla con los requisitos de escala y nivel de información adecuada para transformarlo en un mapa táctil, en este ejemplo se muestra la base utilizada para el mapa de Equilibrio del Carbono (Figura 1).

- Buscar la mejor forma de representar la información a través de diferentes alturas en el relieve, de texturas (Figura 2) que no lleven a confusión, por tanto deben ser muy diferenciables.

- Considerar los símbolos gráficos como letras o números (Figura 3) que se utilizarán y cómo éstos, deben ir distribuidos sobre la superficie del producto cartográfico.

- Visualizar lo diferente que puede ser adaptar un mapa para un usuario en especial o hacer un prototipo que luego será reproducido muchas veces a través del proceso de termoformado con material PVC (Figura 4).

Estos son los considerandos básicos al hacer una cartografía táctil, pero falta reflexionar acerca de las características del usuario de ese material. Algunos aspectos a considerar:

- Curso al que está dirigido el material

- Alumnos ciegos o con baja visión

- Alumnos sordos con o sin experiencia en el uso e interpretación de este material

- Tiempo que se necesita para explorar el mapa

Cada una de estas alternativas puede llevar a tener que realizar un mapa diferente. $\mathrm{O}$ que al menos se necesite que el alumno deba ser guiado para usarlo.

Una vez resueltos todos los aspectos antes mencionados, se procede a confeccionar el mapa táctil. Para estar seguros de que se ha realizado una buena adaptación, es fundamental someter el producto a la evaluación por parte de personas con discapacidad visual (Figura 5) o auditiva, pues muchas veces el adaptador tiene en la retina el mapa visual al examinar el mapa táctil y pasa por alto aspectos que para la persona con discapacidad visual son relevantes, por ejemplo: texturas que al tacto son similares, signografia Braille distribuida sin un criterio cercanía espacial (Figura 3), el concepto que se quiere comunicar no queda claro, entre otros. 


\section{A Quebra do Equilíbrio do Carbono}

0 carbono é essencial ao meio ambiente natural, mas as mudanças no uso do solo podem liberar as reservas de carbono e contribuir para as alterações climáticas.

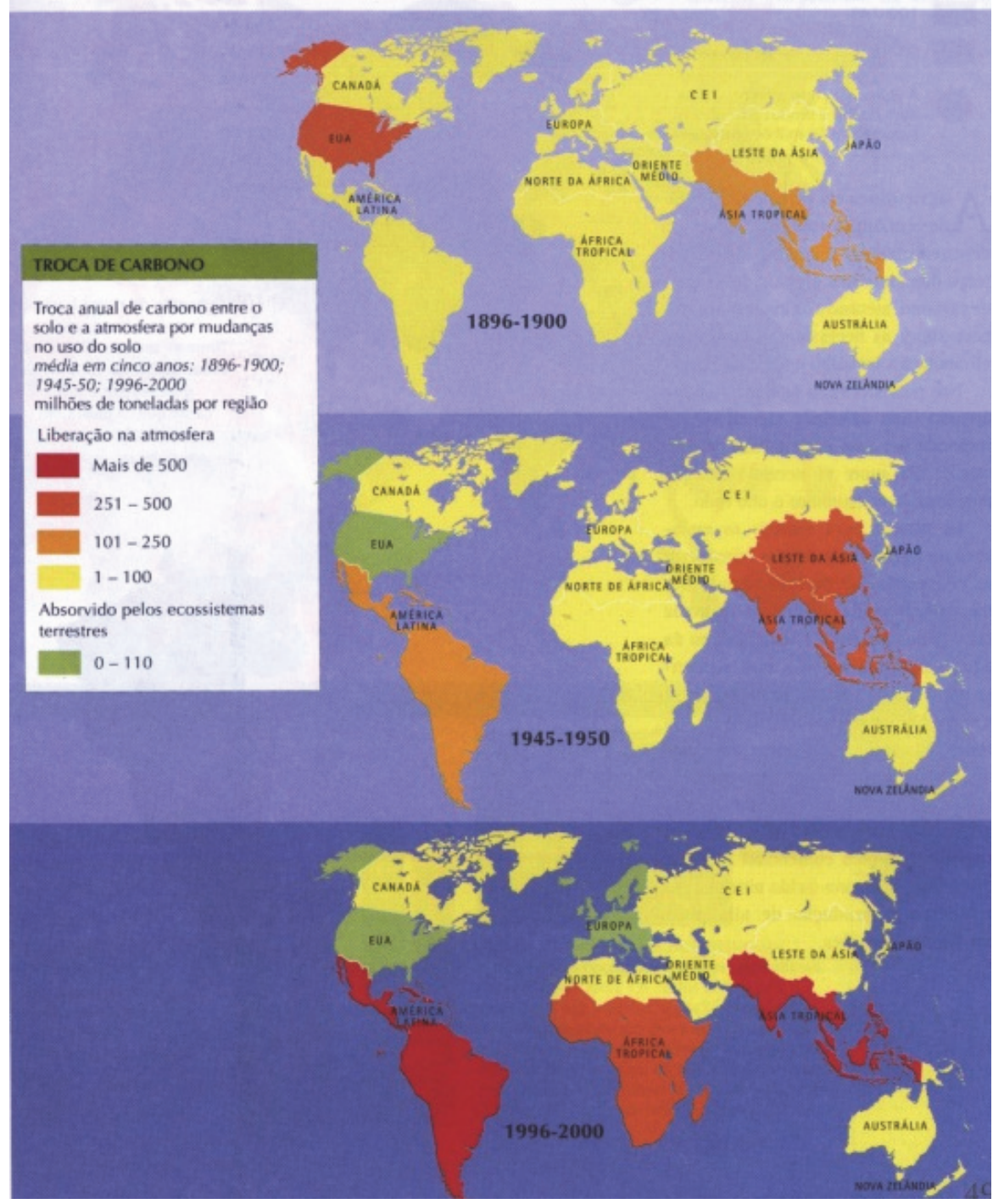

Figura 1. Quiebre equilibrio del carbono.

Fuente: Proyecto IPGH. 

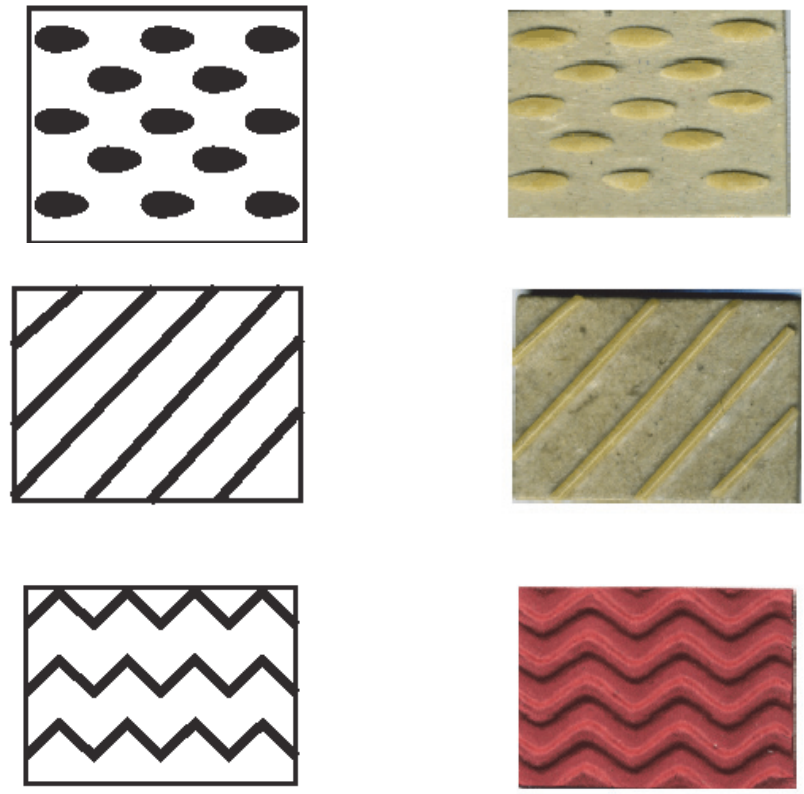

Figura 2. Símbolos por medio de texturas.

Fuente: Informe proyecto IPGH CART.2.1.1.7.2/GEO.2.1.2.9.4.
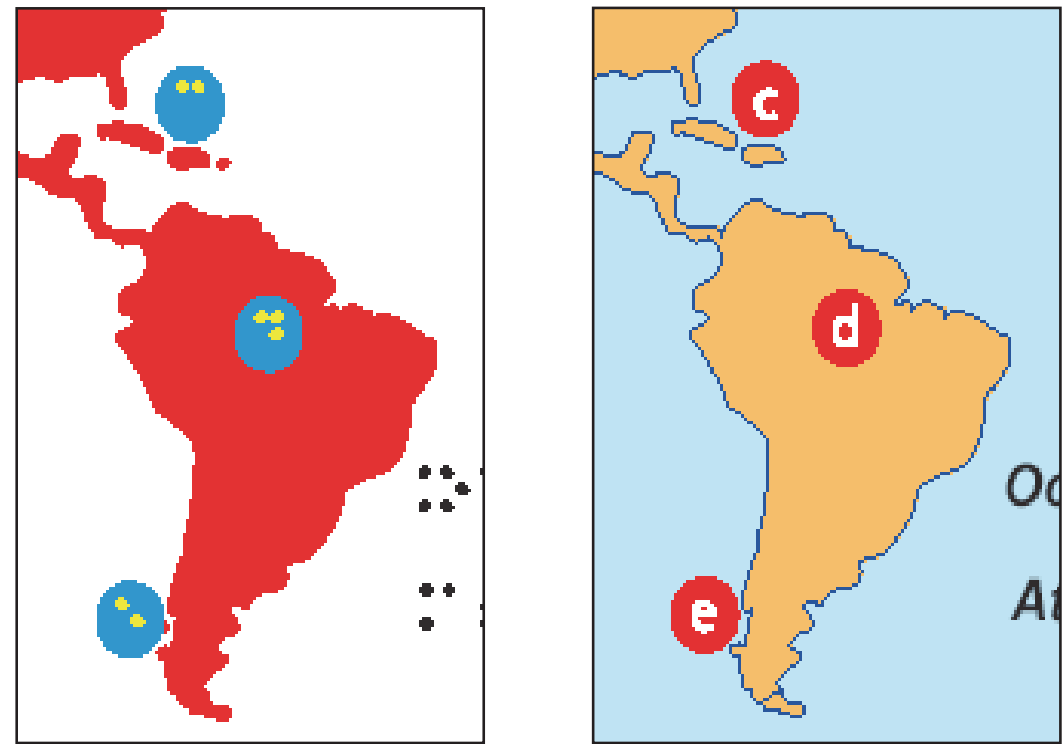

Figura 3. Símbolos gráficos utilizando letras.

Fuente: Proyecto IPGH. 


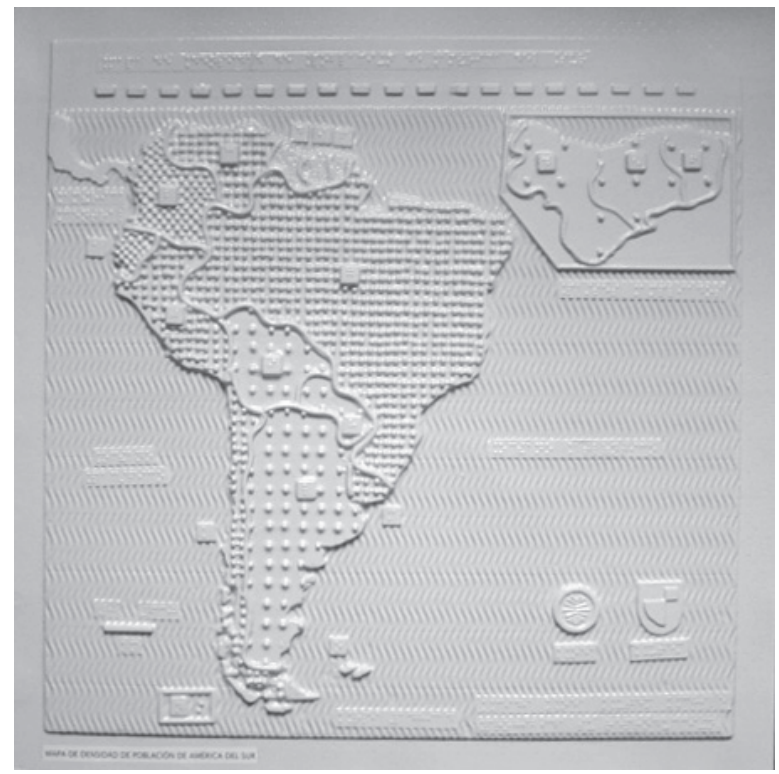

Figura 4. Mapa termoformado densidad población América del Sur. Fuente: Proyecto IPGH.
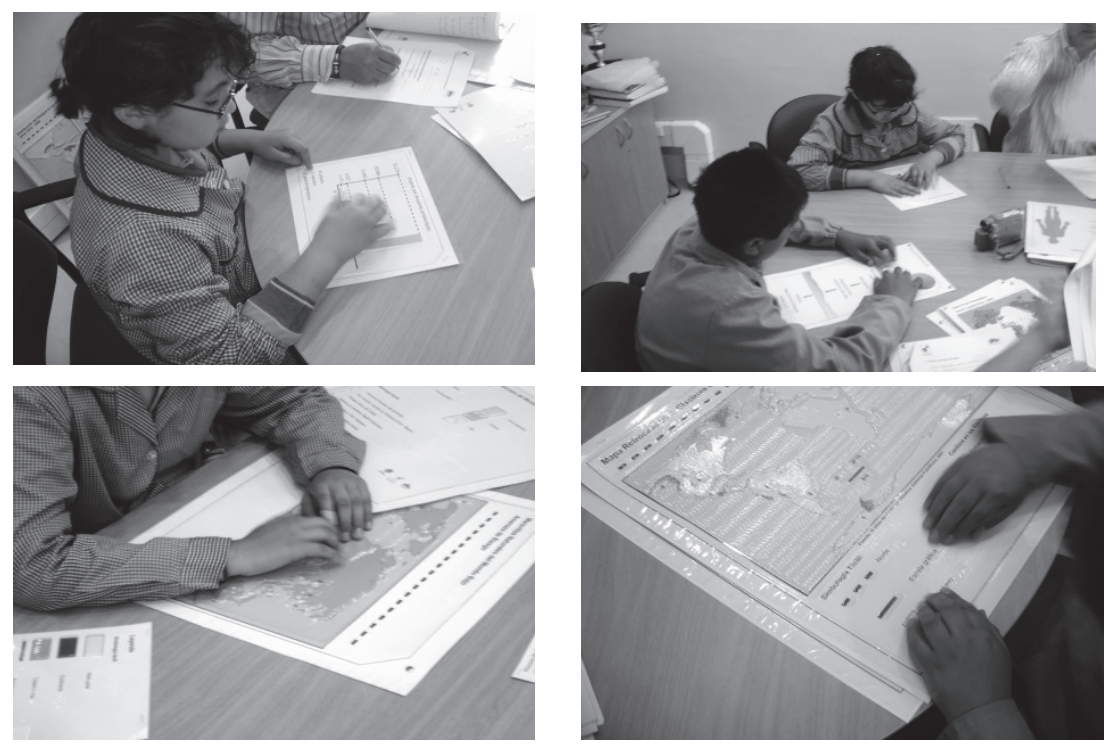

Figura 5. Alumnos ciegos Colegio Santa Lucía evaluando el material. Fuente: Proyecto IPGH. 

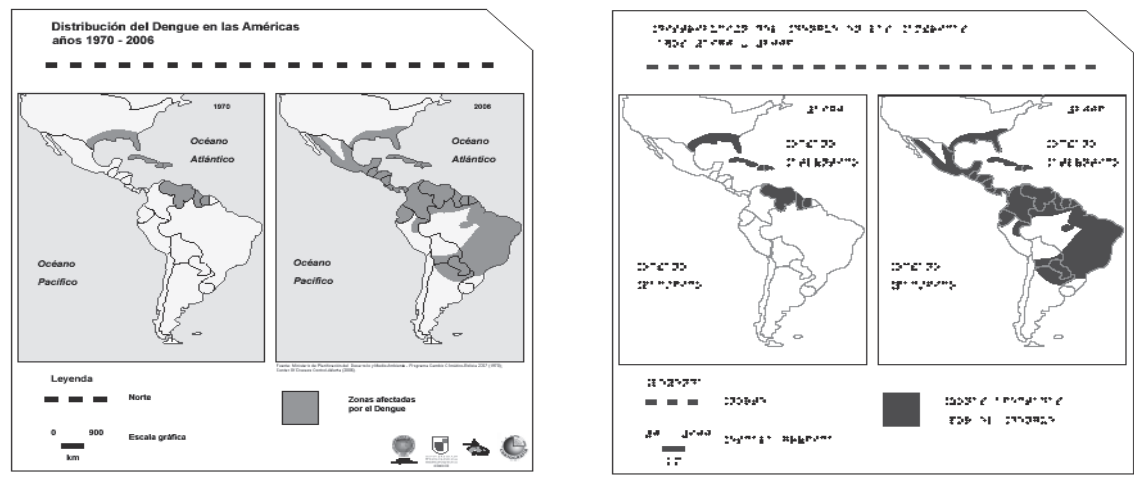

Figura 6. Mapa distribución del dengue en América del Sur en tinta y braille.

Fuente: Proyecto IPGH.

Al desarrollar el proyecto de investigación "Generación de cartografía táctil para la comprensión del calentamiento global y su relación con los eventos naturales" se enfrentaron nuevos desafíos, no sólo había que adaptar mapas sino que cada mapa involucraba representar temas específicos, como Distribución del Dengue en las Américas, (Figura 6) permitiendo también una comparación entre mapas que representan la situación en diferentes años.

En este caso la evaluación debió considerar nuevos aspectos:

- Evaluar si es entendida la simbología del mapa

- Evaluar la comprensión de los temas relacionados con el calentamiento global representados en los mapas y láminas

- Evaluar separadamente los aspectos físicos y conceptuales de la representación involucrados en estos mapas temáticos

\section{Metodología de evaluación del material}

Como ya se mencionó los productos realizados en este proyecto IPGH involucran la comprensión de conceptos como: desertificación, derretimiento de los hielos, cambio del equilibrio del carbono, entre otros, materias que están dentro del currículo educacional, pero como no se tenía la certeza que los alumnos conocieran estas temáticas, fue necesario recurrir al apoyo de los profesores de aula para explicar los fenómenos y conceptos. Tanto en la escuela de ciegos como en la escuela de sordos los profesores hicieron la introducción a los temas.

La evaluación del material, efectuada por los investigadores y ayudantes, fue registrada a través de test, tomas fotográficas y videos, lo cual permite objetivar esta etapa. 


\section{Evaluación con alumnos que presentan discapacidad visual}

Los alumnos que participaron en la evaluación correspondían a una muestra de quinto, sexto y séptimo año básico.

Es relevante mencionar que en este tipo de evaluación grupal las mesas se ordenan en forma de "U" de tal manera que quien explica pueda ir chequeando que los alumnos están haciendo el mismo recorrido sobre el mapa y a la vez puede resolver dudas. Luego cada evaluador se acerca y trabaja con uno a dos alumnos (Figura 7) para hacerles las preguntas del test en un contexto de conversación. Cada alumno tiene su material.

Se ha observado que los alumnos mencionan en voz alta los aspectos que van descubriendo en el material, también hacen preguntas. Lo cual permite evaluar de forma muy dinámica.

Los alumnos con baja visión deben hacer la exploración del material de manera táctil y visual, lo cual les permite utilizar dos canales sensoriales, para identificar y recordar la información.

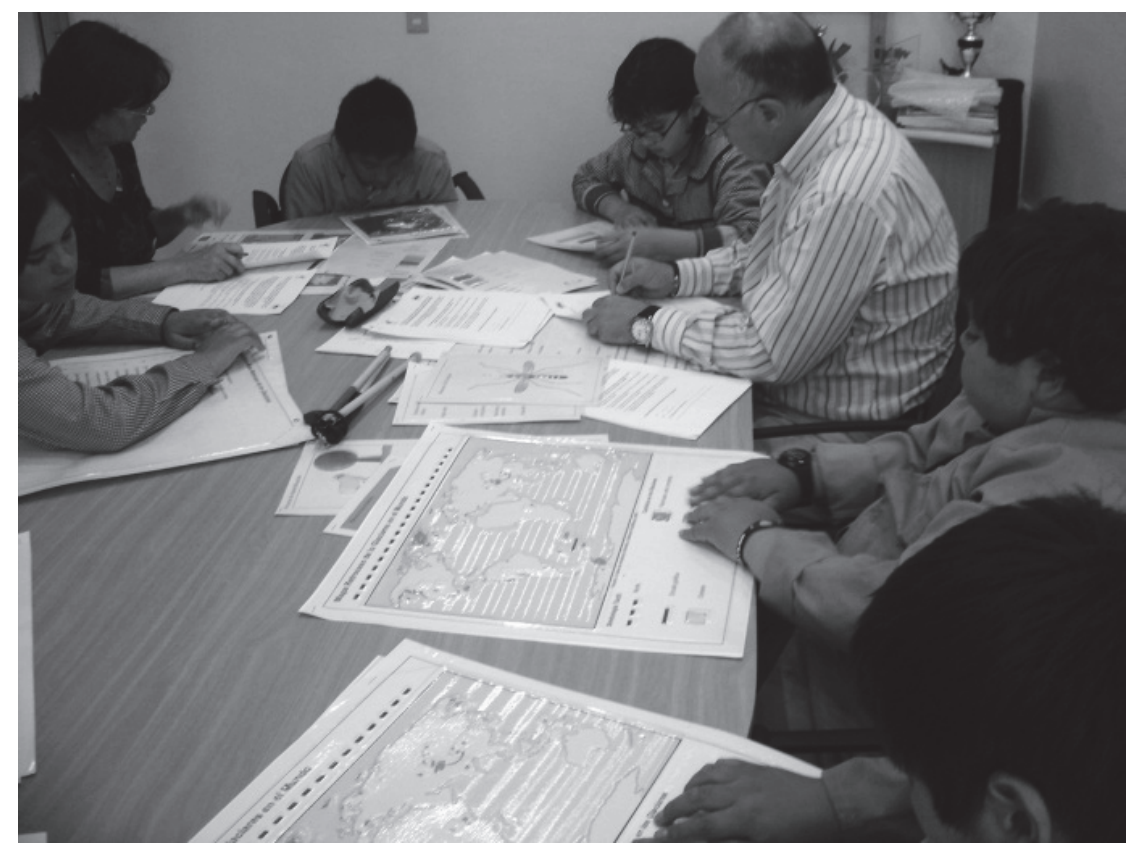

Figura 7. Evaluadores en el momento de hacer las preguntas colegio de ciegos. Fuente: Proyecto IPGH. 


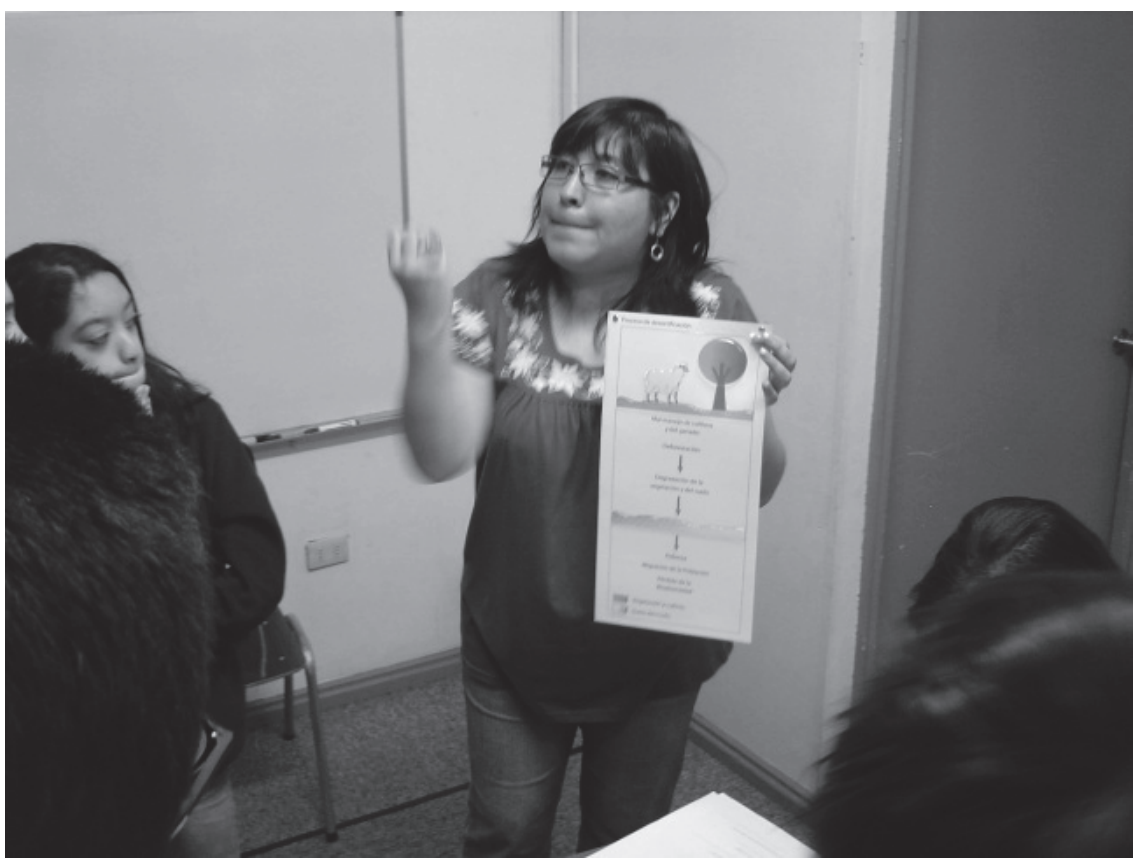

Figura 8. Evaluación en colegio de sordos.

Fuente: Proyecto IPGH.

\section{La evaluación con alumnos que presentan discapacidad auditiva}

Los alumnos que participaron en la evaluación correspondían a quinto y sexto año básico (Figura 8). En este caso la disposición de las mesas es diferente, los alumnos agrupan las mesas, de tal manera que el material quede al centro y ellos se distribuyen alrededor, mientras el profesor explica. Los evaluadores observan y van tomando nota de lo que los estudiantes dicen. ${ }^{1}$

En este caso un set de material (Figura 9) sirve para que todos trabajen, atienden a la explicación y luego exploran el material, lo cual les permite reforzar la información visual que les entregan los colores y simbología del mapa.

Los estudiantes exploran, hacen preguntas a la profesora, comparten sus impresiones con los compañeros, y algunos también relacionan lo que están conociendo con experiencias de vida y noticias.

1 Escuela de Sordos San Francisco de Asís, utiliza el método auditivo-verbal por tanto los alumnos se comunican hablando no por medio del lenguaje de señas. 

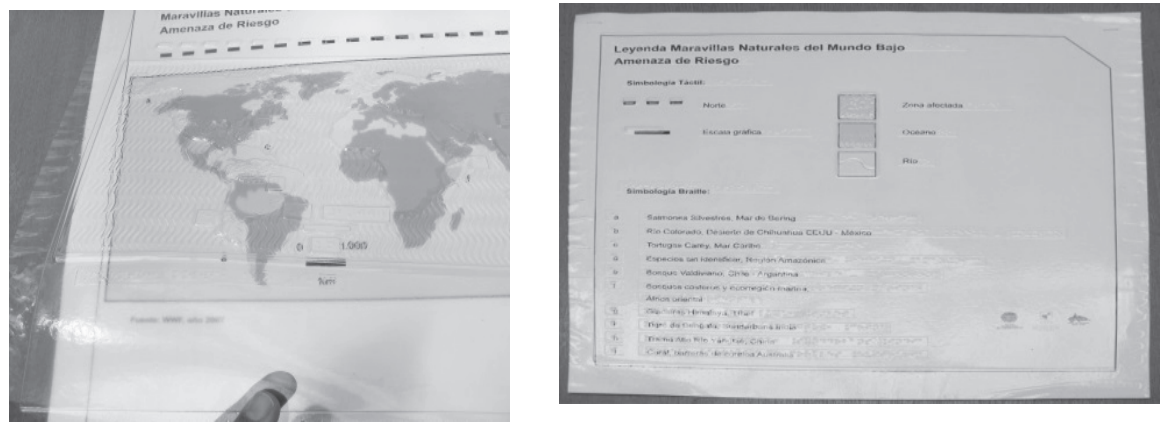

Figura 9. Mapa y leyenda maravillas naturales del mundo bajo amenaza de riesgo, en formato tinta y termoformado transparente.

Fuente: Proyecto IPGH.

Es interesante mencionar que la directora de la Escuela de Sordos San Francisco de Asís, Ximena Vidal, manifiesta que sería importante ampliar el vocabulario de los estudiantes para facilitarles la comprensión de estas temáticas.

\section{Rol del evaluador}

El rol del evaluador en este caso está centrado en la aplicación del test, pues quien está encargado de introducir la temática de cada producto táctil es el profesor del aula, al cual se le entregó un documento pedagógico, una especie de cuadernillo, que contiene información de apoyo sobre las temáticas abordadas, retroceso de los glaciares, proceso de desertificación, efecto invernadero, distribución del dengue, maravillas naturales del mundo y cambios del equilibrio del Carbono.

El evaluador debe ser una persona que conozca los mapas y el test a aplicar, y estar al tanto de algunas estrategias básicas para comunicarse con personas con discapacidad visual y auditiva y tener un rol activo. En el caso de las personas ciegas dar orientación considerando los puntos de referencia y en las personas sordas hablarles mirándolos de frente, entre otras estrategias.

La etapa de evaluación fue desarrollada paralelamente en Argentina, Brasil y Perú, durante el segundo semestre de 2011 en colegios con estudiantes ciegos. En el caso de Chile, se realizó esta evaluación en establecimientos educacionales donde estudiaban alumnos con discapacidad visual y auditiva. Esta evaluación permitió detectar algunos problemas a nivel del reconocimiento táctil y visual. Por otra parte, se pudo determinar si son comprendidos los conceptos del calentamiento global y su manifestación en eventos naturales. 
1. ¿Puede diferenciar las áreas donde hay retroceso de glaciares?

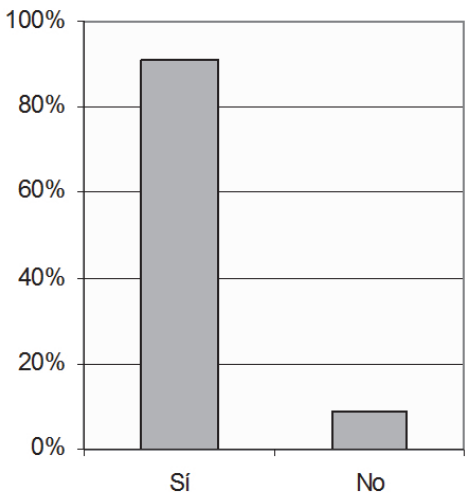

Evaluación alumnos ciegos
1. ¿Puede diferenciar las áreas donde hay retroceso de glaciares?

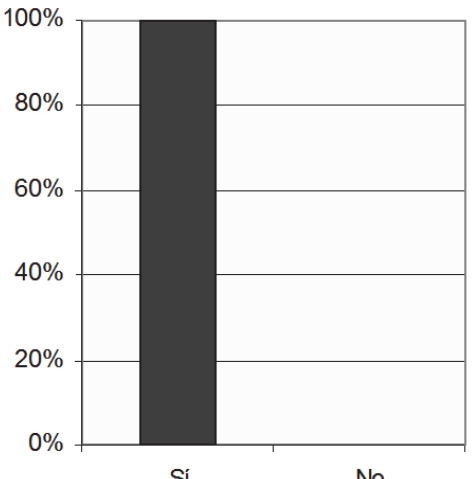

Sí

Evaluación alumnos sordos

Figura 10. Retroceso de los glaciares en el mundo mapa y leyenda maravillas naturales del mundo bajo amenaza de riesgo, en formato tinta y termoformado transparente Fuente: Proyecto IPGH.

\section{Tabulación y representación de los resultados de los test de evaluación}

Tras la aplicación del instrumento de evaluación, se realizan gráficos (Figura 10) teniendo como base esta información que representa a cada uno de los mapas y láminas, separado por colegio y por país. Los gráficos son analizados en forma independiente para cada pregunta, con lo cual se logra comparar los resultados por los distintos colegios y países participantes.

Del análisis de los resultados obtenidos del proceso de evaluación, se obtienen una serie de interesantes comentarios observaciones y sugerencias, que se transforman en la base de las correcciones que se deben hacer en el proceso de optimización del material.

\section{Análisis de los resultados de la evaluación del material}

Para realizar el análisis de los resultados obtenidos, y como una forma de ordenar las conclusiones, se procedió a separar en dos enfoques estos análisis, uno desde lo que se denominó "aspectos físicos de la representación" (ejemplo, norte, texturas, color) y "aspectos conceptuales tratados" (ejemplo, retroceso, desertificación).

Es importante señalar, que la totalidad de los alumnos encuestados, no habían tenido acceso a la información representada, lo cual se transformó en un aporte muy interesante a los estudiantes, quienes comprendieron una serie de fenómenos relacionados con el calentamiento global. 


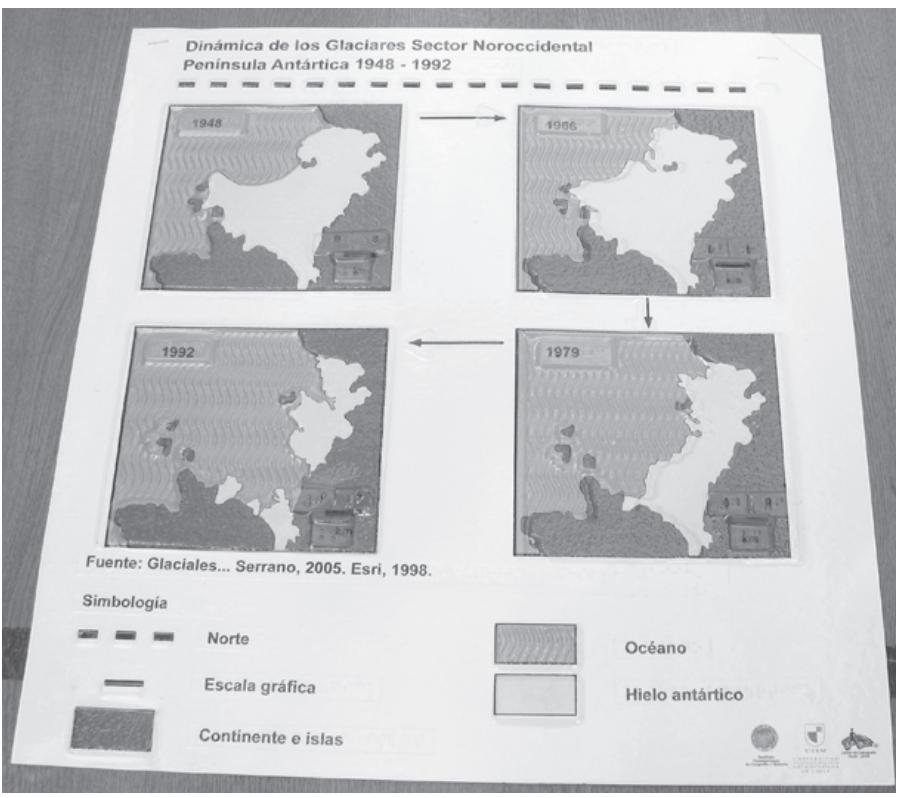

Figura 11. Mapa retroceso de glaciares Península Antártica. Fuente: Proyecto IPGH.
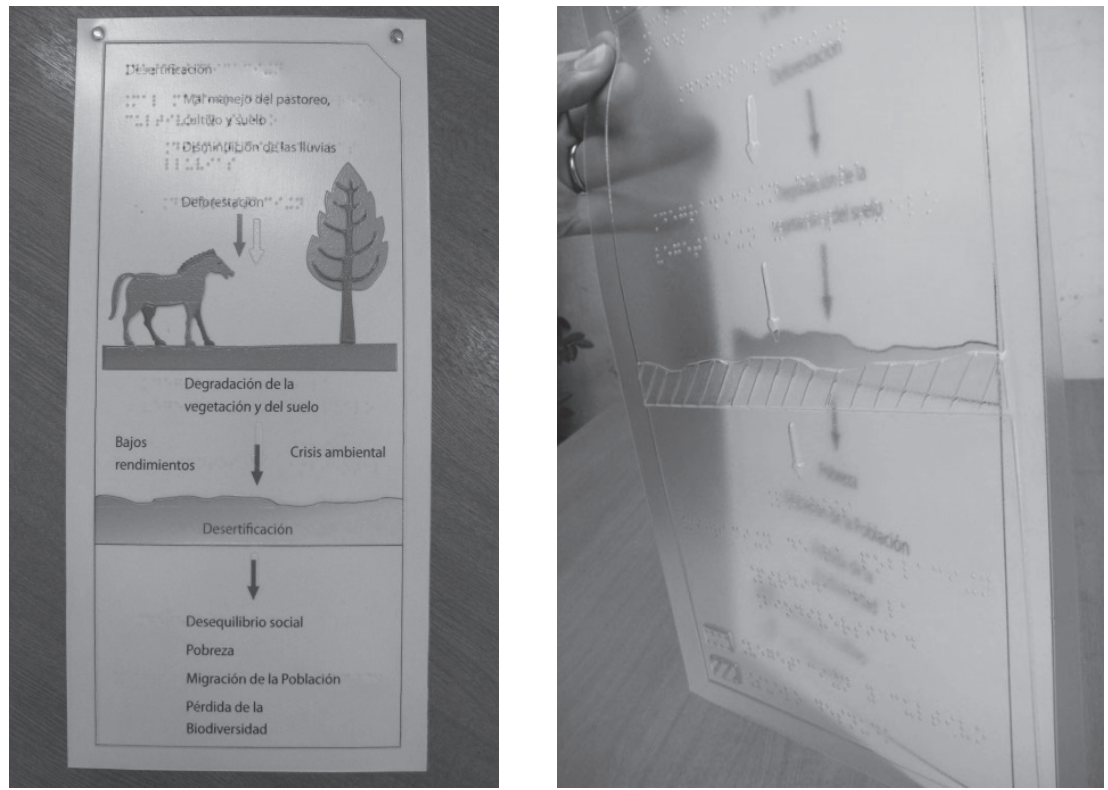

Figura 12. Concepto de desertificación. Fuente: Proyecto IPGH. 


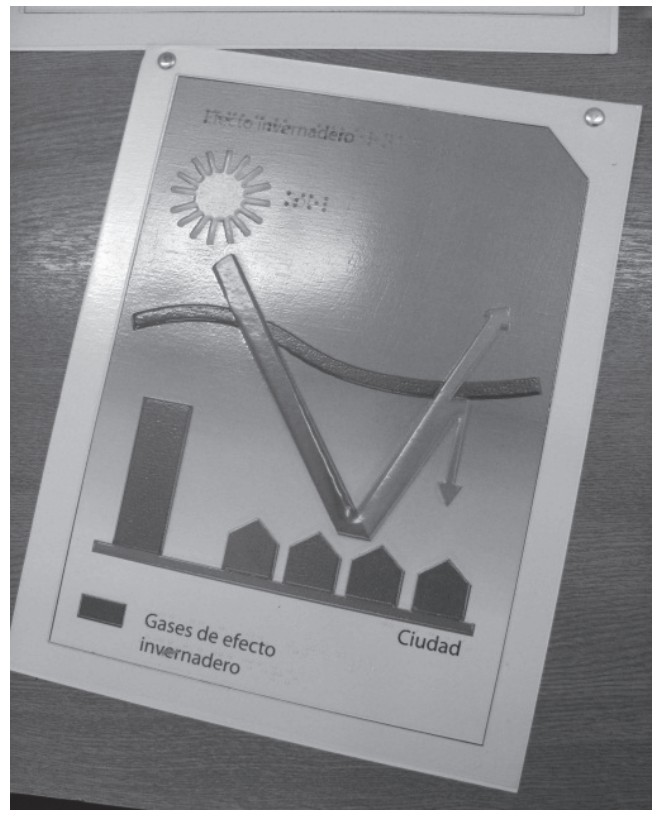

Figura 13. Concepto efecto invernadero.

Fuente: Proyecto IPGH.

\section{Aspectos físicos de la representación}

Los elementos físicos en general recibieron una alta aprobación por parte de los estudiantes evaluadores tanto ciegos como sordos. A continuación se muestran las conclusiones que se señalaron por cada uno de los principales elementos representados:

Norte geográfico: en Brasil, Chile y Perú reconocieron sobre el 90\% la representación del norte geográfico. En cambio en Argentina esta evaluación mostró un menor porcentaje de reconocimiento positivo, ya que sólo el $60 \%$ de los encuestados reconoció esta simbología, esto se puede deber a que anteriormente utilizaban otro símbolo para el norte geográfico.

Mapas y leyendas: la leyenda que acompaña a los mapas es fundamental para que se logre el objetivo de comprender la información, en este caso en promedio, más del $80 \%$ manifestó que la leyenda le permitía comprender la información que estaba representada en el mapa, y que el formato le era adecuado a la simbología presentada y a las dimensiones de ésta.

Texturas: la textura fue un elemento gráfico táctil que se utilizó en los mapas y en los conceptos, donde la información tenía un carácter areal, tanto cualitativo (Re- 
troceso Glaciares Sector Noroccidental Península Antártica 1948-1992), como cuantitativo (Cambio del Equilibrio del Carbono (1896-1900) (1996-2000)) y lineal en la figura del mosquito del Dengue.

Se puede señalar que sobre el $90 \%$ de las texturas utilizadas en las áreas que representaban una variable, fueron reconocidas claramente, con lo cual desde el punto de vista táctil se entendían con facilidad al ser exploradas. Hubo algunas dificultades en el reconocimiento de las líneas utilizadas para representar el mosquito del Dengue, es importante hacer un nuevo diseño.

Color: esta variable visual, fue fundamental para aquellos alumnos que tenían resto de visión y para los alumnos sordos. En general fue muy bien evaluado la utilización del color. Los alumnos con resto de visión opinaron que en el mapa donde se mostraba la liberación de carbono a la atmósfera, se tendía a confundir las tonalidades verdes utilizadas, por lo cual sugieren que se utilice colores con más contraste.

Distribución de varios mapas en una misma línea: este aspecto se consideró fundamental y complejo en el momento de representar cuatro etapas diferentes en distintos períodos de tiempo de una misma variable, como es el mapa de Retroceso de Glaciares en la Península Antártica, (Figura 10) donde los cuatros mapas se distribuyen en una misma lámina. El orden que se definió, fue que el primero se ubicara en el extremo superior izquierdo, luego superior derecho, posteriormente inferior derecho para finalizar con el último mapa en la parte inferior izquierda. Esta secuencia de la dinámica de los glaciares fue comprendida por un universo del $70 \%$.

\section{Aspectos conceptuales de la representación}

Como se mencionó anteriormente, se procedió a evaluar en forma separada la parte conceptual ya que se pretendía con esto, identificar si los alumnos habían percibido más que la representación, es decir el significado del concepto.

Esta comprensión se logró en un muy alto porcentaje sin mayores problemas. En aquellos mapas donde se tenía que percibir los cambios en una superficie (retroceso de un glaciar) se apreció en forma clara este fenómeno, permitiendo incluso trabajar la parte del porcentaje de reducción, lo cual fue muy interesante ya que estaba aplicado a un fenómeno geográfico.

Podemos asegurar que los alumnos al utilizar este material, con la mediación del profesor, perciben de manera eficiente los cambios representados en la cartografía como son expansión, retroceso, distribución, diferenciación, tipos de cambio de un fenómeno que representa el problema del calentamiento global.

Durante la exploración los alumnos logran aplicar conceptos de cantidad tales como más, menos, mayor, menor, igual, los cuales les permiten comprender los procesos de expansión del Dengue, retroceso de los glaciares entre otros. La compa- 
ración del fenómeno en distintos años, permite que los alumnos puedan obtener nuevas deducciones.

En cuanto a la comprensión de un fenómeno tan difícil de entender como es la Liberación del Carbono, fue entendido en muy alto porcentaje, también este tema, permitió hacer preguntas donde se involucraban los continentes, lo cual aportó un objetivo nuevo e interesante, la comprensión del aspecto físico del mundo.

En el momento de relacionar en una figura algún aspecto puntual de un fenómeno específico, se logra con facilidad, esto se observa en el caso del Dengue, donde los alumnos pueden identificar claramente los sectores donde se manifiesta el síntoma del dengue en la figura humana.

La representación de los conceptos (Figura 12) fue un gran aporte a la temáti-ca del calentamiento global, esto permitió la compresión de fenómenos tan específicos como el Efecto Invernadero (Figura 13), donde el color, forma y textura jugaron un papel fundamental. Se hace necesario en el caso de los conceptos contar con una pequeña introducción sobre la materia que se desarrolla.

Considerando todos los productos cartográficos y conceptuales utilizados en la evaluación, podemos afirmar sin error, que este material facilita la elaboración del mapa mental del lugar representado. Cuando tenemos el marco espacial de los contornos, redes de caminos o hidrográfica, límites administrativos, vías de comunicación entre otros, podemos tener representado nuestro mapa mental del mundo.

\section{Conclusión y recomendaciones}

En todo proceso de adaptación del material visual a táctil, se debe incluir obligatoriamente la etapa de evaluación del material, por parte de personas ciegas y sordas, ya que logran percibir aspectos que para las personas que tienen visión no son reconocidos.

El rol que juega el profesor en el aula es fundamental, ya que es el puente entre la temática que se está representando y explicando en los mapas y conceptos y el alumno, sin esta introducción la comprensión se hace más difícil.

Se recomienda filmar la evaluación, con el objetivo de revisar y chequear detalles de la exploración que realizaron los alumnos, la forma cómo se hizo la introducción, la forma cómo se aplicó la pauta de evaluación, el rol que jugó el académico y el evaluador.

La metodología que se está empleando para desarrollar los productos cartográficos, está permitiendo comunicar la problemática ambiental y sus consecuencias en cambios de aspectos geográficos en forma clara, comprendiendo la problemática en sí, el aspecto conceptual que involucra.

La forma de aplicar los test, si se siguen las recomendaciones planteadas, permite generar las instancias más adecuadas para que el resultado sea el óptimo, tenien- 
do en cuenta que no es lo mismo aplicarlas a las personas ciegas que a las personas sordas.

\section{Bibliografía}

Benoit, S. et al. (1993). "La composition des chorèmes dans la modélisation graphique appliquée à La Bourgagne", Mappe Monde, 2, 1993, pp. 37-41.

Coll E., y Barrientos, T. (2003). "Cartografía y tecnología como apoyo al conocimiento geográfico y a la orientación y movilidad de la persona ciega", Proyecto OEA/SEDI/AE/01, Anales V Congreso Latinoamericano de Ciegos (ULAC). Quito, Ecuador.

Maestro, I., (2004). "Fomento de la Movilidad de Invidentes Mediante Cartografía Táctil: criterios de PARTIDA", Revista Internacional de Ciencias de la Tierra.

Pérez, E. (2004). "Los Símbolos Cartográficos Táctiles como elementos de Comunicación de información", Anales de la Sociedad Chilena de Ciencias Geográficas. 